\title{
REMOVABILITY AND NON-INJECTIVITY OF CONFORMAL WELDING
}

\author{
Malik Younsi \\ University of Hawaii at Manoa, Department of Mathematics \\ Honolulu, HI 96822, U.S.A.; malik.younsi@gmail.com
}

\begin{abstract}
We construct a (non-removable) Jordan curve $\Gamma$ and a non-Möbius homeomorphism of the Riemann sphere which is conformal on the complement of $\Gamma$ and maps the curve $\Gamma$ onto itself. The curve is flexible in the sense of Bishop and may be taken to have zero area. The existence of such curves and conformal homeomorphisms is closely related to the non-injectivity of conformal welding.
\end{abstract}

\section{Introduction}

Let $\mathbf{D}$ be the open unit disk, let $\mathbf{D}^{*}:=\widehat{\mathbf{C}} \backslash \overline{\mathbf{D}}$ be the complement of the closed unit disk in the Riemann sphere $\widehat{\mathbf{C}}$, and let $\mathbf{T}:=\partial \mathbf{D}$ be the unit circle. Given a Jordan curve $\Gamma$, let $f: \mathbf{D} \rightarrow \Omega$ and $g: \mathbf{D}^{*} \rightarrow \Omega^{*}$ be conformal maps onto the bounded and unbounded complementary components of $\Gamma$ respectively. Then $f$ and $g$ extend to homeomorphisms on the closure of their respective domains, so that $h_{\Gamma}:=g^{-1} \circ f: \mathbf{T} \rightarrow \mathbf{T}$ defines an orientation-preserving homeomorphism of the unit circle onto itself, called the conformal welding homeomorphism of $\Gamma$.

Note that $h_{\Gamma}$ is uniquely determined by $\Gamma$ up to pre- and post-composition by automorphisms of the unit disk. Moreover, if $T$ is a Möbius transformation, then $\Gamma$ and $T(\Gamma)$ have the same conformal welding homeomorphism, so that the conformal welding correspondence

$$
\mathcal{W}:[\Gamma] \mapsto\left[h_{\Gamma}\right]
$$

is well-defined, from the family of Jordan curves, modulo Möbius equivalence, to the family of orientation-preserving homeomorphisms of the circle, modulo pre- and post-composition by automorphisms of the disk.

This correspondence between Jordan curves and circle homeomorphisms has appeared over the years to be of central importance in a wide variety of areas of mathematics and applications, such as Teichmüller theory, Kleinian groups, computer vision and numerical pattern recognition [6,30], and so forth. For more information on the applications of conformal welding, the interested reader may consult the survey article [9]. We also mention that recent years have witnessed a strong renewal of interest in conformal welding as other variants and generalizations have been introduced and developed, such as generalized conformal welding [4, 8], random conformal welding [1], conformal welding for finitely connected regions [20], conformal welding of random surfaces [29] and conformal laminations of trees [21, 27], including applications to Shabat polynomials and Grothendieck's dessins d'enfants. Conformal laminations are also related to the recent groundbreaking work of Miller and Sheffield on the relationship between the Brownian map and Liouville Quantum Gravity [22, 23, 24].

https://doi.org/10.5186/aasfm.2018.4322

2010 Mathematics Subject Classification: Primary 30C35; Secondary 37E10, 30C85.

Key words: Conformal welding, removability, flexible curves, capacity. 
It is well-known that the conformal welding correspondence $\mathcal{W}$ is not surjective; in other words, there are orientation-preserving homeomorphisms of the circle (even analytic everywhere except at one point) which are not conformal welding homeomorphisms. On the other hand, every quasisymmetric homeomorphism $h: \mathbf{T} \rightarrow \mathbf{T}$ is the conformal welding of some Jordan curve (in this case, a quasicircle). Here quasisymmetric means that adjacent $\operatorname{arcs} I, J \subset \mathbf{T}$ of equal length are mapped by $h$ onto arcs of comparable length:

$$
M^{-1} \leq \frac{|h(I)|}{|h(J)|} \leq M
$$

The fact that every such $h$ is a conformal welding homeomorphism is usually referred to as the fundamental theorem of conformal welding and was first proved by Pfluger [26] in 1960. Another proof based on quasiconformal mappings was published shortly after by Lehto and Virtanen [17]. See also the papers of Bishop [4] and Schippers and Staubach [28]. We also mention that other sufficient conditions for a given circle homeomorphism $h$ to be a welding were obtained by Lehto [16] and Vainio [31], in terms of $h$ being sufficiently "nice". In Section 2, we recall a deep theorem of Bishop [4] saying that on the other hand, any "wild" enough $h$ is also the welding of some Jordan curve. Finding a complete characterization of conformal welding homeomorphisms is most likely a very difficult problem.

This paper, however, deals with the (non) injectivity of the welding correspondence $\mathcal{W}$. Recall that if $\Gamma$ is a Jordan curve, then $T(\Gamma)$ has the same welding homeomorphism $h_{\Gamma}$, for any Möbius transformation $T$. Are these the only curves with this property?

If $\Gamma$ and $\widetilde{\Gamma}$ are two Jordan curves such that $h_{\Gamma}=h_{\widetilde{\Gamma}}$, then there are conformal maps $f: \mathbf{D} \rightarrow \Omega, g: \mathbf{D}^{*} \rightarrow \Omega^{*}, \widetilde{f}: \mathbf{D} \rightarrow \widetilde{\Omega}, \widetilde{g}: \mathbf{D}^{*} \rightarrow \widetilde{\Omega}^{*}$ such that

$$
g^{-1} \circ f=\widetilde{g}^{-1} \circ \widetilde{f}
$$

on $\mathbf{T}$, i.e.

$$
\widetilde{g} \circ g^{-1}=\tilde{f} \circ f^{-1}
$$

on $\Gamma$, where $\widetilde{\Omega}, \widetilde{\Omega}^{*}$ are the bounded and unbounded complementary components of the curve $\widetilde{\Gamma}$. It follows that the map $F: \widehat{\mathbf{C}} \rightarrow \widehat{\mathbf{C}}$ defined by

$$
F(z)= \begin{cases}\left(\tilde{f} \circ f^{-1}\right)(z) & \text { if } z \in \Omega \cup \Gamma, \\ \left(\widetilde{g} \circ g^{-1}\right)(z) & \text { if } z \in \Omega^{*}\end{cases}
$$

is a homeomorphism conformal on $\widehat{\mathbf{C}} \backslash \Gamma$ which maps the curve $\Gamma$ onto the curve $\widetilde{\Gamma}$. This shows that if $\Gamma$ is conformally removable, then $\Gamma$ uniquely corresponds to its conformal welding homeomorphism $h_{\Gamma}$, up to Möbius equivalence.

Definition 1.1. We say that a compact set $E \subset \mathbf{C}$ is conformally removable if every $F \in \mathrm{CH}(E)$ is Möbius, where $\mathrm{CH}(E)$ is the collection of homeomorphisms $F: \widehat{\mathbf{C}} \rightarrow \widehat{\mathbf{C}}$ which are conformal on $\widehat{\mathbf{C}} \backslash E$.

Single points, smooth curves and more generally, sets of $\sigma$-finite length, are all conformally removable. On the other hand, the theory of quasiconformal mappings can be used to prove that sets of positive area are never conformally removable. The converse is well-known to be false, and there exist nonremovable sets (even Jordan curves) of Hausdorff dimension one [3, 15] and removable sets of Hausdorff dimension two [18, Chapter V, Section 3.7]. In fact, no geometric characterization of conformally removable sets is known. We also mention that this notion of removability appears 
naturally in the study of various important problems in Complex Analysis and related areas, such as Koebe's uniformization conjecture [10, 33] and the MLC conjecture on the local connectivity of the Mandelbrot set $[11,12,13]$. See also the survey article [32] for more information.

Now, recall that if a Jordan curve $\Gamma$ is conformally removable, then $\Gamma$ uniquely corresponds to its conformal welding homeomorphism, modulo Möbius equivalence. The starting point of this paper is the following question.

Question 1.2. Does the converse hold? Namely, if $\Gamma$ is a non-removable Jordan curve, does there necessarily exist another curve having the same welding homeomorphism, but which is not a Möbius image of $\Gamma$ ?

Several papers in the literature seem to imply that the answer is trivially yes, either without proof or with the following argument:

If $\Gamma$ is not removable, then there exists a non-Möbius $F \in \mathrm{CH}(\Gamma)$. But then the curve $F(\Gamma)$ has the same welding homeomorphism as $\Gamma$, and is not Möbius equivalent to it, since $F$ is not a Möbius transformation.

See e.g. [25, Lemma 2], [14, Corollary II.2], [8, Section 4], [2, Corollary 1], [3, p. 324-325], [9, Section 3], [4, Remark 2], [1, Section 2.3], [19, Corollary 1.4].

Although it is true and easy to see that $\Gamma$ and $F(\Gamma)$ have the same welding homeomorphism, it is not clear at all that these two curves are not Möbius equivalent, since there could a priori exist a Möbius transformation $T$ such that $F(\Gamma)=T(\Gamma)$, even though $F$ itself is not Möbius. As far as we know, this remark first appeared in Fortier Bourque's Master's Thesis [7]. The question of whether such $\Gamma, F$ and $T$ as above actually exist was, however, left open. In this paper, we answer that question in the affirmative.

Theorem 1.3. There exists a Jordan curve $\Gamma$ and a non-Möbius homeomorphism $F: \widehat{\mathbf{C}} \rightarrow \widehat{\mathbf{C}}$ conformal on $\widehat{\mathbf{C}} \backslash \Gamma$ such that $F(\Gamma)=\Gamma$. Moreover, the curve $\Gamma$ may be taken to have zero area.

The construction is based on a result of Bishop [4] characterizing the conformal welding homeomorphisms of so-called flexible curves.

Theorem 1.3 shows that the above argument claiming to answer Question 1.2 is in fact incorrect, and whether removability really characterizes injectivity of conformal welding remains unknown.

The remainder of the paper is organized as follows. In Section 2, we recall Bishop's characterization of the conformal welding homeomorphisms of flexible curves. Then, in Section 3, we use this result to prove Theorem 1.3. In Section 4, we discuss the non-injectivity of conformal welding for curves of positive area. Finally, Section 5 contains some open problems related to Question 1.2.

\section{Flexible curves and log-singular homeomorphisms}

We first need the definition of logarithmic capacity, following [4]. For $E \subset \mathbf{T}$ Borel, let $\mathcal{P}(E)$ denote the collection of all Borel probability measures on $E$.

Definition 2.1. Let $\mu \in \mathcal{P}(E)$.

(i) The energy of $\mu$, noted $I(\mu)$, is given by

$$
I(\mu):=\iint \log \frac{2}{|z-w|} d \mu(z) d \mu(w) .
$$


(ii) The logarithmic capacity of $E$, noted $\operatorname{cap}(E)$, is defined as

$$
\operatorname{cap}(E):=\frac{1}{\inf \{I(\mu): \mu \in \mathcal{P}(E)\}} .
$$

It is well-known that logarithmic capacity is nonnegative, monotone and countably subadditive (see e.g. [5]). We will also need the simple fact that bi-Hölder homeomorphisms of the circle preserve sets of zero capacity.

We can now define log-singular homeomorphisms.

Definition 2.2. Let $I, J$ be two subarcs of the unit circle. An orientationpreserving homeomorphism $h: I \rightarrow J$ is log-singular if there exists a Borel set $E \subset I$ such that both $E$ and $h(I \backslash E)$ have zero logarithmic capacity.

The following inductive construction of log-singular homeomorphisms was outlined in [4, Remark 9]. We reproduce it here for the reader's convenience.

Proposition 2.3. Let $I, J$ be two subarcs of $\mathbf{T}$. Then there exists a log-singular homeomorphism $h: I \rightarrow J$.

Proof. Start with any orientation-preserving linear homeomorphism $h_{1}: I \rightarrow J$. At the first step, divide $I$ into two subarcs, denoted by red and blue respectively, in such a way that the red subarc has small logarithmic capacity, say less than $2^{-1}$. Now, define a homeomorphism $h_{2}$ on $I$ which is linear on the red subarc and the blue subarc, and which satisfies $h_{2}(I)=h_{1}(I)=J$. We also construct $h_{2}$ such that it maps the blue subarc onto an arc of logarithmic capacity also less than $2^{-1}$.

Now, at the $n$-th step, suppose that $I$ has been divided into a finite number of $\operatorname{arcs}\left\{I^{k, n}\right\}$, and that we have a homeomorphism $h_{n}: I \rightarrow J$ which is linear on each of those arcs. First, divide each $I^{k, n}$ into $n$ arcs of equal length $I_{1}^{k, n}, I_{2}^{k, n}, \ldots, I_{n}^{k, n}$. Then, divide each $I_{j}^{k, n}$ into a red and a blue subarc, in such a way that the union of all the red subarcs has logarithmic capacity less than $2^{-n}$. Now, define a homeomorphism $h_{n+1}: I \rightarrow J$ which is linear on each of the red and blue subarcs, and which satisfies $h_{n+1}\left(I_{j}^{k, n}\right)=h_{n}\left(I_{j}^{k, n}\right)$ for each $j, k$. We also construct $h_{n+1}$ so that the union of all the images of the blue subarcs under the map has logarithmic capacity less than $2^{-n}$.

It is not difficult to see that these maps $h_{n}$ converge to an orientation-preserving homeomorphism $h: I \rightarrow J$. Indeed, if $\epsilon>0$, then we can choose $N$ sufficiently large so that for $n \geq N$, the $\operatorname{arcs} h_{n}\left(I_{j}^{k, n}\right)$ all have length less than $\epsilon$. If $m \geq n$ and $x \in I$, then $x$ belongs to one of the $\operatorname{arcs} I_{j}^{k, n}$ and $h_{m}(x)$ belongs to $h_{n}\left(I_{j}^{k, n}\right)$ by construction, thus

$$
\left|h_{n}(x)-h_{m}(x)\right|<\epsilon .
$$

This shows that the sequence $\left(h_{n}\right)$ is uniformly Cauchy and therefore has a continuous limit $h: I \rightarrow J$, which has to be an orientation-preserving homeomorphism, by construction.

Finally, the map $h: I \rightarrow J$ is log-singular. Indeed, if $E$ is the set of points in $I$ which belong to infinitely many of the red subarcs, then

$$
\operatorname{cap}(E) \leq \sum_{n \geq m} 2^{-n} \quad(m \in \mathbf{N})
$$

by the subadditivity of logarithmic capacity, so that $\operatorname{cap}(E)=0$. On the other hand, we have

$$
h(\mathbf{T} \backslash E) \subset \bigcup_{m=1}^{\infty} \bigcap_{n \geq m} h_{n+1}\left(B_{n}\right),
$$


where $B_{n}$ is the union of all the blue subarcs constructed at the $n$-th step. It follows that $h(\mathbf{T} \backslash E)$ is contained in a countable union of sets of zero logarithmic capacity, so that $\operatorname{cap}(h(\mathbf{T} \backslash E))=0$, again by the subadditivity of logarithmic capacity.

We will also need the following definition.

Definition 2.4. A Jordan curve $\Gamma \subset \mathbf{C}$ is a flexible curve if the following two conditions hold:

(i) Given any Jordan curve $\widetilde{\Gamma}$ and $\epsilon>0$, there exists $F \in \mathrm{CH}(\Gamma)$ such that

$$
d(F(\Gamma), \widetilde{\Gamma})<\epsilon
$$

where $d$ is the Hausdorff distance.

(ii) Given points $z_{1}, z_{2}$ in each complementary component of $\Gamma$ and points $w_{1}, w_{2}$ in each complementary component of $\widetilde{\Gamma}$, we can choose $F$ above so that $F\left(z_{1}\right)=w_{1}$ and $F\left(z_{2}\right)=w_{2}$.

One can think of a flexible curve $\Gamma$ as being "highly" non-removable, in the sense that $\mathrm{CH}(\Gamma)$ is very large. Examples of flexible curves with any Hausdorff dimension between 1 and 2 were constructed in [3].

There is a close relationship between flexible curves and log-singular homeomorphisms. Indeed, Bishop proved in [4] that an orientation-preserving homeomorphism $h: \mathbf{T} \rightarrow \mathbf{T}$ is log-singular if and only if it is the conformal welding of a flexible curve $\Gamma$. In particular, this implies that every such $h$ is the conformal welding of a dense family of curves, since if $F \in \mathrm{CH}(\Gamma)$ is as in Definition 2.4, then $h$ is also the conformal welding homeomorphism of $F(\Gamma)$. We shall actually need the following stronger result, see [4, Theorem 25].

Theorem 2.5. (Bishop) Let $h: \mathbf{T} \rightarrow \mathbf{T}$ be an orientation-preserving log-singular homeomorphism with $h(1)=1$, and let $f_{0}, g_{0}$ be two conformal maps of $\mathbf{D}$ and $\mathbf{D}^{*}$ respectively onto disjoint domains. Then for any $0<r<1$ and any $\epsilon>0$, there are conformal maps $f$ and $g$ of $\mathbf{D}$ and $\mathbf{D}^{*}$ onto the two complementary components of a Jordan curve $\Gamma$ satisfying the following conditions:

(i) $h=g^{-1} \circ f$ on $\mathbf{T}$;

(ii) $\left|f(z)-f_{0}(z)\right|<\epsilon$ for all $z \in \mathbf{D}$ with $|z| \leq r$;

(iii) $\left|g(z)-g_{0}(z)\right|<\epsilon$ for all $z \in \mathbf{D}^{*}$ with $|z| \geq 1 / r$;

Moreover, the maps $f, g$ may be constructed such that $f(1)=g(1)=\infty$ and such that the curve $\Gamma$ has zero area.

Remark. Since the last part of Theorem 2.5 was not stated explicitly in [4], let us briefly explain how it follows from the construction. First, since $h(1)=1$, condition (i) implies that $f(1)=g(1)$. Composing $f$ and $g$ by a Möbius transformation $T$ if necessary, we can assume that $f(1)=g(1)=\infty$. Note that if $f$ and $g$ approximate $T^{-1} \circ f_{0}$ and $T^{-1} \circ g_{0}$ on compact subsets of $\mathbf{D}$ and $\mathbf{D}^{*}$ respectively, then $T \circ f$ and $T \circ g$ approximate $f_{0}$ and $g_{0}$.

Now, to see that the curve $\Gamma$ can be taken to have zero area, note that the main part of the proof in [4] is to construct quasiconformal mappings $f: \mathbf{D} \rightarrow \Omega$ and $g: \mathbf{D} \rightarrow \Omega^{*}$ onto the complementary components of a Jordan curve $\Gamma$ satisfying conditions (i), (ii) and (iii), and having quasiconstants close to 1 . These maps $f$ and $g$ are obtained as limits of quasiconformal mappings $f_{n}: \mathbf{D} \rightarrow \Omega_{n}$ and $g_{n}: \mathbf{D}^{*} \rightarrow \Omega_{n}^{*}$ onto smooth Jordan domains with disjoint closures, with $\infty \in \Omega_{n}^{*}$. By construction, the curve $\Gamma$ is contained in the topological annulus $A_{n}:=\widehat{\mathbf{C}} \backslash\left(\Omega_{n} \cup \Omega_{n}^{*}\right)$, for each $n$. Moreover, the domains $\Omega_{n}$ and $\Omega_{n}^{*}$ are of the form $\Omega_{n}:=F_{n}(t \mathbf{D})$ and $\Omega_{n}^{*}:=G_{n}\left(\mathbf{D}^{*} / t\right)$, 
where $t<1$ is sufficiently close to 1 and $F_{n}, G_{n}$ are quasiconformal mappings of $\mathbf{D}, \mathbf{D}^{*}$ onto the complementary components of a smooth Jordan curve $\Gamma_{n}$. Clearly, by taking $t$ closer to 1 if necessary, we can assume that the topological annulus $A_{n}$ has area as small as we want, say less than $2^{-n}$. Then the curve $\Gamma$ will have zero area.

Finally, the last part of the proof is to apply the measurable Riemann mapping theorem to replace $f$ and $g$ by conformal maps $\Phi \circ f$ and $\Phi \circ g$, where $\Phi$ is a quasiconformal mapping of the sphere. Since $\Phi$ preserves sets of area zero and since it can be assumed to fix $\infty$ by composing with a Möbius transformation, the new conformal maps can also be taken so that they send 1 to $\infty$ and map $\mathbf{T}$ onto a curve of zero area.

\section{Conformal homeomorphisms fixing a curve}

We can now prove Theorem 1.3. Recall that we want to construct a Jordan curve $\Gamma$ and a non-Möbius homeomorphism $F: \widehat{\mathbf{C}} \rightarrow \widehat{\mathbf{C}}$ conformal on $\widehat{\mathbf{C}} \backslash \Gamma$ such that $F(\Gamma)=\Gamma$.

The idea of the construction is the following. Suppose that such a curve $\Gamma$ and such a non-Möbius homeomorphism $F \in \mathrm{CH}(\Gamma)$ exist, and suppose that $F$ preserves the orientation of $\Gamma$. Let $f: \mathbf{D} \rightarrow \Omega$ and $g: \mathbf{D}^{*} \rightarrow \Omega^{*}$ be conformal maps onto the two complementary components of the curve. Then $F \circ f$ and $F \circ g$ are also conformal maps of $\mathbf{D}$ and $\mathbf{D}^{*}$ onto $\Omega$ and $\Omega^{*}$ respectively, so that

$$
F \circ f=f \circ \sigma
$$

and

$$
F \circ g=g \circ \tau
$$

for some $\sigma, \tau \in \operatorname{Aut}(\mathbf{D})$. Note that neither $\sigma$ nor $\tau$ is the identity, since otherwise $F$ would also be the identity, contradicting the fact that it is not Möbius. Now, since $F$ is continuous on $\Gamma$, we must have

$$
f \circ \sigma \circ f^{-1}=g \circ \tau \circ g^{-1}
$$

there, which can be rewritten as

$$
W \circ \sigma=\tau \circ W
$$

on $\mathbf{T}$, where $W:=h_{\Gamma}$ is the conformal welding of $\Gamma$. We thus obtain a functional equation for the welding of the curve.

The strategy now is to proceed backward. Start with an orientation-preserving homeomorphism $W: \mathbf{T} \rightarrow \mathbf{T}$ satisfying the functional equation (1) for some $\sigma, \tau \in$ Aut(D). Suppose in addition that we can construct $W$ so that it is the conformal welding homeomorphism of some Jordan curve $\Gamma$, i.e. $W=g^{-1} \circ f$ where $f$ and $g$ are conformal maps of $\mathbf{D}$ and $\mathbf{D}^{*}$ respectively onto the two complementary components $\Omega, \Omega^{*}$ of $\gamma$. Then we can define a map $F$ conformal on both sides of $\gamma$ by

$$
F(z)= \begin{cases}\left(f \circ \sigma \circ f^{-1}\right)(z) & \text { if } z \in \Omega, \\ \left(g \circ \tau \circ g^{-1}\right)(z) & \text { if } z \in \Omega^{*}\end{cases}
$$

and the fact that $W=g^{-1} \circ f$ satisfies Equation (1) implies that $F$ extends to a homeomorphism of the whole sphere. Clearly, the map $F \in \mathrm{CH}(\Gamma)$ fixes the curve $\Gamma$, and all that remains to prove is that we can choose $\sigma, \tau$ and $W$ such that $F$ is not a Möbius transformation.

The main difficulty here is that if we choose $W$ to be sufficiently nice, e.g. quasisymmetric, so that it is a conformal welding homeomorphism, then the curve $\Gamma$ 
will be removable and $F$ will necessarily be Möbius. In order to circumvent this difficulty, a promising approach is to construct log-singular homeomorphic solutions of the functional equation (1).

Lemma 3.1. Let $a, b>0$, and let $\phi$ be a Möbius transformation mapping the upper half-plane $\mathbf{H}$ onto the open unit disk $\mathbf{D}$ with $\phi(\infty)=1$, say

$$
\phi(z):=\frac{z-i}{z+i}
$$

Define $\widetilde{\sigma}, \widetilde{\tau} \in \operatorname{Aut}(\mathbf{H})$ by $\widetilde{\sigma}(z):=z+a$ and $\widetilde{\tau}(z):=z+b$, and set

$$
\sigma:=\phi \circ \widetilde{\sigma} \circ \phi^{-1}
$$

and

$$
\tau:=\phi \circ \widetilde{\tau} \circ \phi^{-1}
$$

so that $\sigma, \tau \in \operatorname{Aut}(\mathbf{D})$. Then there exists a log-singular orientation-preserving homeomorphism $W: \mathbf{T} \rightarrow \mathbf{T}$ which satisfies

$$
W \circ \sigma=\tau \circ W
$$

Proof. Let $I_{0}:=\phi([0, a))$ and $J_{0}:=\phi([0, b))$, and let $W: I_{0} \rightarrow J_{0}$ be a logsingular orientation-preserving homeomorphism, as in Proposition 2.3. For $n \in \mathbf{Z}$, set $I_{n}:=\sigma^{n}\left(I_{0}\right)$ and $J_{n}:=\tau^{n}\left(J_{0}\right)$. Note that the subarcs $I_{n}$ are pairwise disjoint and that

$$
\bigcup_{n \in \mathbf{Z}} I_{n}=\mathbf{T} \backslash\{1\}
$$

and similarly for the $J_{n}$ 's. Now, extend $W$ to all of $\mathbf{T}$ by setting

$$
W(z):=\left(\tau^{n} \circ W \circ \sigma^{-n}\right)(z) \quad\left(z \in I_{n}\right)
$$

and $W(1):=1$. Clearly, the map $W: \mathbf{T} \rightarrow \mathbf{T}$ thereby obtained is an orientationpreserving homeomorphism. Moreover, if $z \in I_{n}$ for some $n$, then $\sigma(z) \in I_{n+1}$, so that

$$
\begin{aligned}
W(\sigma(z)) & =\left(\tau^{n+1} \circ W \circ \sigma^{-(n+1)}\right)(\sigma(z)) \\
& =\left(\tau \circ\left(\tau^{n} \circ W \circ \sigma^{-n}\right)\right)(z)=(\tau \circ W)(z),
\end{aligned}
$$

which shows that $W$ satisfies Equation (1).

It remains to prove that $W: \mathbf{T} \rightarrow \mathbf{T}$ satisfies the log-singular condition. Let $E_{0} \subset I_{0}$ such that $\operatorname{cap}\left(E_{0}\right)=0$ and $\operatorname{cap}\left(W\left(I_{0} \backslash E_{0}\right)\right)=0$. For $n \in \mathbf{Z}$, let $E_{n}:=$ $\sigma^{n}\left(E_{0}\right) \subset I_{n}$. Note that $\operatorname{cap}\left(E_{n}\right)=0$ for all $n$, since bi-Hölder homeomorphisms preserve sets zero logarithmic capacity. It follows that $E:=\bigcup_{n} E_{n}$ also has capacity zero, by subadditivity. Finally, we have

$$
\begin{aligned}
W(\mathbf{T} \backslash E) & =\bigcup_{n \in \mathbf{Z}} W\left(I_{n} \backslash E_{n}\right) \cup\{1\}=\bigcup_{n \in \mathbf{Z}} W\left(\sigma^{n}\left(I_{0} \backslash E_{0}\right)\right) \cup\{1\} \\
& =\bigcup_{n \in \mathbf{Z}} \tau^{n}\left(W\left(I_{0} \backslash E_{0}\right)\right) \cup\{1\},
\end{aligned}
$$

which shows that $\operatorname{cap}(W(\mathbf{T} \backslash E))=0$, again by subadditivity and the fact that bi-Hölder homeomorphisms preserve sets of zero logarithmic capacity.

This completes the proof of the lemma.

We can now proceed with the proof of Theorem 1.3. 
Proof. Let $a, b>0$ with $a \neq b$, and let $W: \mathbf{T} \rightarrow \mathbf{T}, \sigma$ and $\tau$ be as in Lemma 3.1, so that

$$
W \circ \sigma=\tau \circ W
$$

Also, let $0<\epsilon<|a-b| / 4$, let $z_{1}, z_{2}$ be points in the upper half-plane $\mathbf{H}$ and lower half-plane $\mathbf{H}^{*}$ respectively, and let $K_{1} \subset \mathbf{H}$ and $K_{2} \subset \mathbf{H}^{*}$ be compact sets such that $z_{1}, z_{1}+a \in K_{1}$ and $z_{2}, z_{2}+b \in K_{2}$. Lastly, take $0<r<1$ sufficiently close to 1 so that $\phi\left(K_{1}\right) \subset \mathbf{D}(0, r)$ and $\phi\left(K_{2}\right) \subset \mathbf{C} \backslash \mathbf{D}(0,1 / r)$, where $\phi$ is the Möbius transformation of Lemma 3.1.

By Theorem 2.5, we can write $W:=g^{-1} \circ f$, where $f$ and $g$ are conformal maps of $\mathbf{D}$ and $\mathbf{D}^{*}$ onto the two complementary components $\Omega$ and $\Omega^{*}$ of a Jordan curve $\Gamma$, such that $f(1)=g(1)=\infty$,

$$
\left|f(z)-\phi^{-1}(z)\right|<\epsilon \quad(|z| \leq r)
$$

and

$$
\left|g(z)-\phi^{-1}(z)\right|<\epsilon \quad(|z| \geq 1 / r)
$$

In particular, the above inequalities hold for $z \in \phi\left(K_{1}\right)$ and $z \in \phi\left(K_{2}\right)$ respectively.

Now, define a map $F$ by

$$
F(z)= \begin{cases}\left(f \circ \sigma \circ f^{-1}\right)(z) & \text { if } z \in \Omega \\ \left(g \circ \tau \circ g^{-1}\right)(z) & \text { if } z \in \Omega^{*}\end{cases}
$$

so that $F$ is conformal on $\widehat{\mathbf{C}} \backslash \Gamma$. Also, the equation $W \circ \sigma=\tau \circ W$ on $\mathbf{T}$ is equivalent to

$$
f \circ \sigma \circ f^{-1}=g \circ \tau \circ g^{-1}
$$

on $\Gamma$, so that $F$ extends to a homeomorphism of $\widehat{\mathbf{C}}$. Clearly, this map satisfies $F(\Gamma)=\Gamma$.

It remains to prove that $F$ is not a Möbius transformation. Suppose, in order to obtain a contradiction, that $F$ is Möbius. First, note that $F(\infty)=\infty$, so that $F$ has to be linear, say $F(z)=c z+d$. Now, the map $F$ can be rewritten as

$$
F(z)= \begin{cases}\left(\tilde{f} \circ \tilde{\sigma} \circ \tilde{f}^{-1}\right)(z) & \text { if } z \in \Omega, \\ \left(\widetilde{g} \circ \widetilde{\tau} \circ \widetilde{g}^{-1}\right)(z) & \text { if } z \in \Omega^{*},\end{cases}
$$

where $\tilde{f}:=f \circ \phi: \mathbf{H} \rightarrow \Omega, \widetilde{g}:=g \circ \phi: \mathbf{H}^{*} \rightarrow \Omega^{*}$, and $\widetilde{\sigma}(z):=z+a, \widetilde{\tau}(z):=z+b$ are as in Lemma 3.1. It is easy to see from this that $F$ has only one fixed point, at infinity, so that $c=1$.

Now, note that for $z \in \mathbf{H}$, we have

$$
f(\phi(z))+d=\widetilde{f}(z)+d=F(\widetilde{f}(z))=(\tilde{f} \circ \widetilde{\sigma})(z)=\widetilde{f}(z+a)=f(\phi(z+a)),
$$

so that in particular,

$$
d-a=f\left(\phi\left(z_{1}+a\right)\right)-f\left(\phi\left(z_{1}\right)\right)-a=f\left(\phi\left(z_{1}+a\right)\right)-\left(z_{1}+a\right)-f\left(\phi\left(z_{1}\right)\right)+z_{1} .
$$

By Inequality (2) with $z$ replaced by $\phi\left(z_{1}\right), \phi\left(z_{1}+a\right) \in \phi\left(K_{1}\right)$, we get

$$
|d-a| \leq \epsilon+\epsilon=2 \epsilon \text {. }
$$

Similarly, using the formula for $F$ on $\Omega^{*}$ and Inequality (3), we get

$$
|d-b| \leq 2 \epsilon
$$

and combining the two inequalities yields

$$
|a-b| \leq|a-d|+|d-b| \leq 4 \epsilon,
$$


which contradicts the choice of $\epsilon$. It follows that $F$ is not a Möbius transformation.

Finally, we can take $\Gamma$ to have zero area, by Theorem 2.5. This completes the proof of Theorem 1.3.

\section{Non-injectivity of conformal welding for curves of positive area}

In this section, we mention that the argument described in the introduction, although incorrect in general, can nonetheless be made to work in the case of curves with positive area.

Theorem 4.1. If $\Gamma$ is a Jordan curve with positive area, then there is another curve having the same welding homeomorphism, but which is not a Möbius image of $\Gamma$.

The idea of the proof is quite simple. Since $\Gamma$ has positive area, it is in particular not removable, so there is a non-Möbius homeomorphism $F: \widehat{\mathbf{C}} \rightarrow \widehat{\mathbf{C}}$ which is conformal off $\Gamma$. As already mentioned, the curve $F(\Gamma)$ has the same welding as $\Gamma$, thus is a good candidate for the curve we want to construct. Unfortunately, as we saw in Section 3, it may happen that this curve is a Möbius image of the original one, even though $F$ itself is not Möbius.

However, in the positive-area case, it is easy to see using the measurable Riemann mapping theorem that the collection of non-Möbius elements of $\mathrm{CH}(\Gamma)$ is infinitedimensional, in some sense. A dimension argument relying on Ahlfors-Bers and Brouwer's Invariance of Domain can then be applied to conclude that there must be at least one non-Möbius $F \in \mathrm{CH}(\Gamma)$ such that $F(\Gamma) \neq T(\Gamma)$ for every Möbius transformation $T$.

As far as we know, Theorem 4.1 was first stated by Katznelson, Nag and Sullivan in [14]. See [7, Theorem 4.22] for a complete and detailed proof. It would be very interesting to find a more constructive proof though.

\section{Concluding remarks}

In view of Theorem 4.1, Question 1.2 can be reduced to the following.

Question 5.1. If $\Gamma$ is a non-removable Jordan curve with zero area, does there necessarily exist another curve having the same conformal welding homeomorphism, but which is not a Möbius image of $\Gamma$ ?

As observed in Section 4, the size of $\mathrm{CH}(\Gamma)$ may be relevant here.

Question 5.2. If $\Gamma$ is a non-removable Jordan curve with zero area, is the collection of non-Möbius elements of $\mathrm{CH}(\Gamma)$ necessarily large, or infinite-dimensional, in some sense?

If $\Gamma$ is non-removable, then there exists at least one non-Möbius homeomorphism $F: \widehat{\mathbf{C}} \rightarrow \widehat{\mathbf{C}}$ conformal off $\Gamma$, but as far as we know, it is still open in general whether there must exist another non-Möbius element of $\mathrm{CH}(\Gamma)$ which is not of the form $T \circ F$, for $T$ Möbius.

Finally, we conclude by mentioning that a positive answer to Question 5.1 would follow if one could prove that there always exists a non-Möbius homeomorphism of $\widehat{\mathbf{C}}$ conformal off $\Gamma$ which maps $\Gamma$ onto a curve of positive area.

Acknowledgments. The author thanks Chris Bishop and Don Marshall for helpful discussions. 


\section{References}

[1] Astala, K., P. Jones, A. Kupiainen, and E. Saksman: Random conformal welding. - Acta Math. 207, 2011, 203-254.

[2] Bishop, C. J.: Conformal welding of rectifiable curves. - Math. Scand. 67, 1990, 61-72.

[3] Bishop, C. J.: Some homeomorphisms of the sphere conformal off a curve. - Ann. Acad. Sci. Fenn. Ser. A I Math. 19, 1994, 323-338.

[4] Bishop, C. J.: Conformal welding and Koebe's theorem. - Ann. of Math. (2) 166, 2007, 613656.

[5] Carleson, L.: Selected problems on exceptional sets. - D. Van Nostrand Co., Inc., Princeton, N.J.-Toronto, 1997.

[6] Ebenfelt, P., D. Khavinson, and H. S. Shapiro: Two-dimensional shapes and lemniscates. - Complex analysis and dynamical systems IV. Part 1, 553, 2011, 45-59.

[7] Fortier Bourque, M.: Applications quasiconformes et soudure conforme. - Masters Thesis, Université Laval, 2010.

[8] Hamilton, D. H.: Generalized conformal welding. - Ann. Acad. Sci. Fenn. Ser. A I Math. 16, 1991, 333-343.

[9] Hamilton, D. J.: Conformal welding. - Handbook of complex analysis: geometric function theory 1, 2002, 137-146.

[10] He, Z.-X., and O. Schramm: Rigidity of circle domains whose boundary has $\sigma$-finite linear measure. - Invent. Math. 115, 1994, 297-310.

[11] Kahn, J.: Holomorphic removability of Julia sets. - arXiv:math/9812164, 1998.

[12] KAhn, J., and M. Lyubich: The quasi-additivity law in conformal geometry. - Ann. of Math. (2) 169, 2009, 561-593.

[13] Kahn, J., and M. Lyubich: Local connectivity of Julia sets for unicritical polynomials. - Ann. of Math. (2) 170, 2009, 413-426.

[14] Katznelson, Y., S. NAG, and D. Sullivan: On conformal welding homeomorphisms associated to Jordan curves. - Ann. Acad. Sci. Fenn. Ser. A I Math. 15, 1990, 293-306

[15] Kaufman, R.: Fourier-Stieltjes coefficients and continuation of functions. - Ann. Acad. Sci. Fenn. Ser. A I Math. 9, 1984, 27-31.

[16] Lehto, O.: Homeomorphisms with a prescribed dilatation. - Lecture Notes in Math. 118, $1968,58-73$.

[17] Lehto, O., and K. I. Virtanen: On the existence of quasiconformal mappings with prescribed complex dilatation. - Ann. Acad. Sci. Fenn. Ser. A I Math. 274, 1960, 1-24.

[18] Lehto, O., and K. I. Virtanen: Quasiconformal mappings in the plane. - Springer-Verlag, New York-Heidelberg, 1973.

[19] Lind, J., and S. RohdE: Space-filling curves and phases of the Loewner equation. - Indiana Univ. Math. J. 61, 2012, 2231-2249.

[20] Marshall, D. E.: Conformal welding for finitely connected regions. - Comput. Methods Funct. Theory 11, 2011, 655-669.

[21] Marshall, D. E., and S. Rohde: The zipper algorithm for conformal maps and the computation of Shabat polynomials and dessins. - In preparation.

[22] Miller, J., and S. Sheffield: Liouville quantum gravity and the Brownian map I: the QLE(8/3,0) metric. - arXiv:1507.00719, 2015.

[23] Miller, J., and S. Sheffield: Liouville quantum gravity and the Brownian map II: geodesics and continuity of the embedding. - arXiv:1605.03563, 2016.

[24] Miller, J., and S. Sheffield: Liouville quantum gravity and the Brownian map III: the conformal structure is determined. - arXiv:1608.05391, 2016. 
[25] OiKaWA, K.: Welding of polygons and the type of Riemann surfaces. - Kōdai Math. Sem. Rep. $13,1961,37-52$.

[26] Pfluger, A.: Ueber die Konstruktion Riemannscher Flächen durch Verheftung. - J. Indian Math. Soc. (N.S.) 24, 1960, 401-412.

[27] Rohde, S.: Conformal laminations and Gehring trees. - Preprint.

[28] Schippers, E., and W. Staubach: A symplectic functional analytic proof of the conformal welding theorem. - Proc. Amer. Math. Soc. 143, 2015, 265-278.

[29] Sheffield, S.: Conformal weldings of random surfaces: SLE and the quantum gravity zipper. - Ann. Probab. 44, 2016, 3474-3545.

[30] Sharon, E., and D. Mumford: 2d-shape analysis using conformal mapping. - 2004 IEEE Computer Society Conference on Computer Vision and Pattern Recognition 2, 2004, 350-357.

[31] VAinio, J. V.: Conditions for the possibility of conformal sewing. - Ann. Acad. Sci. Fenn. Ser. A I Math. Diss. 53, 1985, 1-43.

[32] Younsi, M.: On removable sets for holomorphic functions. - EMS Surv. Math. Sci. 2, 2015, $219-254$.

[33] Younsi, M.: Removability, rigidity of circle domains and Koebe's conjecture. - Adv. Math. 303, 2016, 1300-1318.

Received 3 March 2017 • Accepted 12 September 2017 